\title{
Designing a risk management framework for forecasting national security issues
}

\author{
S. Meyer-Nieberg, S. Pickl \& M. Zsifkovits \\ Institut für Theoretische Informatik, Mathematik und \\ Operations Research, Fakultät für Informatik, \\ Universität der Bundeswehr München, Germany
}

\begin{abstract}
Detecting and identifying future risks is a major issue for public decision makers, especially in the field of national security. Decision makers need to identify threats in order to be able to react to them adequately and so reduce risks. Therefore, we established a general risk management support guideline for public decision makers with a focus on national security. The framework aims at identifying future risks, analyzing and evaluating them, so that concrete actions can be set that tackle the potential threat. The risk management framework is thereby based on the core process of the ISO31000 risk management norm. Therefore we are combining several techniques and tools from the field of Operations Research (OR) to guide the decision maker through the risk management core process (risk identification, risk analysis, risk evaluation). Especially in the field of risk identification, the collection of high quality data is a key issue as this data is the foundation for the further creation of possible future scenarios. Therefore, the risk identification faces up to six individual steps of analysis, including qualitative and quantitative OR methods. Several tools were developed that enable a country-based screening for developing threats as well as an internet-based topic monitoring. Out of the concluding catalogue of hazards possible future scenarios are designed, analyzed, and ranked according to their probability of occurrence and importance. Finally, these scenarios are evaluated using a variety of quantitative OR methods, simulation models (mainly $\mathrm{ABM}$ and SD), or Fuzzy Logic Analysis.

Keywords: risk management, risk management process, operations research, OR, ISO31000.
\end{abstract}




\section{Introduction}

The modern world is interconnected on a broad scale making it difficult to predict future developments (Habegger [8]). Especially in the security and military domain, decision makers are facing significant change. (Buch [2]). However, an early detection and analysis of developing threats in the area of national security plays a major role. Strategic foresight is often cited as a tool to inform public policy and decision makers of potential future developments decreasing the chance of harmful surprises. While it stems from the economic sector it has been recently applied more and more in the area of national security (Habegger [8]).

In this paper, we introduce a risk management framework based on the ISO31000 norm for the area of public policy that is designed to take potential future risks into account.

\section{Risk management process: the ISO31000 norm}

Risk management is the means by which the policy maker keeps risks for humans and their livelihoods as low as possible, or at least within acceptable bounds. This leads to the question of where the level of acceptance can be set. These aspects make the risk management process even more complex and lead to the need of comparisons and planning from a holistic perspective (Federal Office for Civil Protection, 2014: 4).

Therefore, a structured guideline through the process of risk management might be helpful to cope with future risks, especially in the public sector with its huge spectrum of uncertainties. There is a standardized risk management process structure available, called ISO3100 norm. This generalized process guideline is a promising framework for such a structured process. According to the definition of the ISO31000 risk management process, it is structured as shown in Figure 1 (ISO31000, 2009).

At its core, the process guides through the identification of risks, their analysis, and finally their evaluation. These three steps are the basis for the resulting risk treatment. However, before starting with the risk identification, the decision maker needs to establish the context of the field of interest. The risk management core process is furthermore accompanied by "Communication and Consultation" and "Monitoring and Review" as interactive tasks that need to be handled by the decision maker continuously.

As we identified the risk management core process as being ideal for the support of several approaches from the field of Operations Research (OR), we created a framework that supports the decision maker and guides through the management core process based on several OR approaches and tools. However, one has to note that the presented and recommended tools need to be selected, analyzed and evaluated for every case separately. Thus, the process needs adaption for each application that might be evaluated. However, the basic structure stays unchanged. 


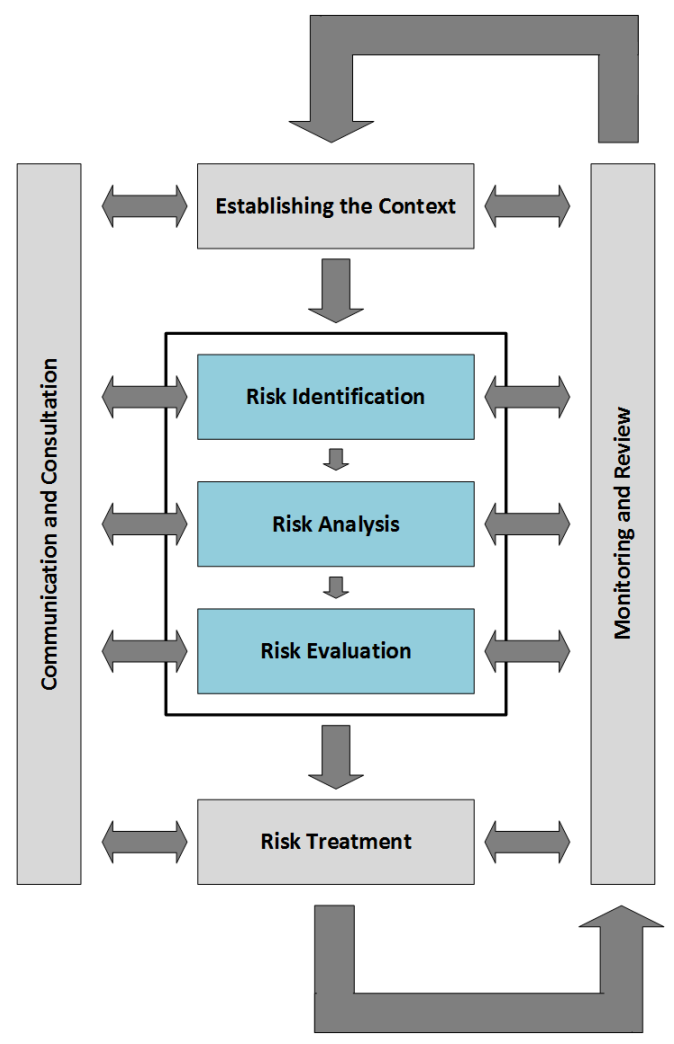

Figure 1: $\quad$ ISO risk management process (ISO31000, 2009).

In general, the process is based on the earlier established context of interest and leads to concrete recommendations for responding to the risk. For the general context we estimated issues from national security and military interventions.

\section{The risk management framework}

As explained above, we concentrate on establishing a framework to support decision makers in the risk management process in its core tasks: risk identification (RI), risk analysis (RA), and risk evaluation (RE) (as shown in Figure 2).

In the core process, each task is a single unit and needs to be completed before the next step can be handled, as the information from each task is the basis for the following. The documentation of tasks and their findings as well as the transfer of knowledge across several steps thereby play a major role in the overall process. In the military domain these tasks are generally known as "risk assessment". 


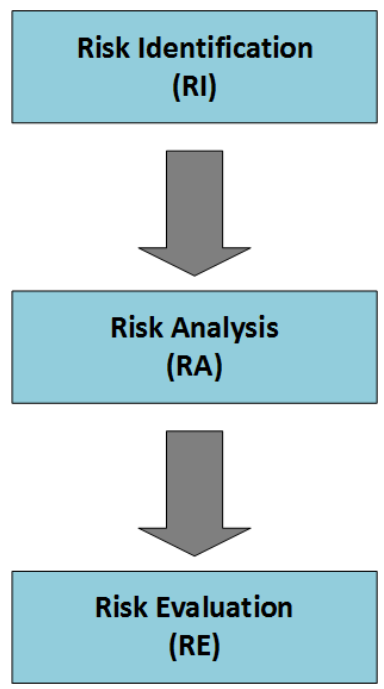

Figure 2: Core tasks in the risk management process.

In the upcoming section the three steps of the risk management core process are explained in more detail and several tools are presented for future use and combination in one overall risk management framework.

\subsection{Risk identification: catalogue of hazards}

After establishing the general context one has to define the problem area itself and analyze it in detail. The objective of this step is to collect and structure as much hazard data as possible in order to create therewith a catalogue of hazards. The catalogue of hazards thereby describes, which threats are existing in the predefined future time horizon. Especially for the field of national security the consideration of several existing qualitative and quantitative methods is a promising approach for finding areas of interest. Originally, foresight focused on only one particular area, e.g. science and technology, health, or environment (Habegger [8]) neglecting potential interconnections between different sectors. However, restricting the analysis to a single sector may present a risk in itself if the area of national security and public policy is concerned. The risk management process must therefore take several areas into account and must select the particular focus points very carefully. Therefore, qualitative and quantitative methods for future analysis have been identified and analyzed in a project conducted at the Universität der Bundeswehr München and supported by the Planungsamt of the German Federal Armed Forces. The aim is to gain general insight into existing and potential future risks. These first insights and data are of great importance, as the further analysis is based on those findings (such as the definition of keywords, prioritization of regions, etc.). Interviews with experts might give first ideas of potential threats. Additionally, internet 
searches, scientific or technical publications, social media may alert the analyst to potential developments. While single signals may not appear as important, the coincidence of several signals from different sectors may represent a key issue for the organization. Here, however human judgment is required. Therefore, the information gained has to be analyzed by experts with respect to underlying emerging issues. After the area of interest has been narrowed, an issued-centered scanning is recommended (Amanatidou et al. [1]). In this paper, we propose to use a combination of several approaches, such as qualitative and quantitative OR methods, an early warning tool and a tool for internet based topic monitoring, both developed at the Universität der Bundeswehr Munich, as well as Google trends analysis. However, the ideal combination of tools depends on the prior defined context. Finally, the used tools should give information for the catalogue of hazards, whereby the use of expert interviews or focus groups is recommended for the final step in this phase.

Concerning national security, it is worthwhile to observe a country's social and economic developments. Several organizations provide indicators describing a country's properties over time. The time-series can be used a) to analyze the past and current situation and b) to predict the future development. The early warning tool developed provides thus an indicator-based approach, where indicators can be chosen with respect to the area of interest and emerging issues. Examples might be the infant mortality rate which is one of the key factors in Goldstone's model on political instability. In some cases, new, specially designed, indicators will have to be used. For many purposes, however, the set of country-based indicators that is provided by the World Bank may already offer sufficient information: The set of indicators spans several areas ranging from data concerning agricultural and rural development over health, education, and poverty to urban development. The early warning tool allows a structured integration into a risk management process. The country-based tool enables the visualization of the past development of indicators and a prediction into the future by making use of quantitative methods for future analysis (Masala and Pickl [11]). Furthermore, explanatory models, as for example the model in (Goldstone et al. [7]) which addresses the political instability of states are provided. The modular tool enables the analyst to detect geographical areas where e.g. political instabilities are more likely to come up in the nearer future than in others. An example is shown in Figure 3.

The internet is one of the vastest sources of data and information today, containing blogs, discussion forums, news sites as well as dedicated databases. Data can take many forms ranging from multimedia to text files and can be presented in several formats. The use of the internet or more correctly the use of Web Mining techniques in Future Research has gained more and more momentum in recent years (Palomino et al. [13]). Therefore, a topic monitoring tool was developed. The tool is able to cope with multilingual information and comprises three components: a keyword-based internet search, an analysis component, and a user interface for visualization. The analyst defines high-level keywords that are automatically translated into several languages and the web is screened on articles about this issue (Stutzki [14]). The actual search is 

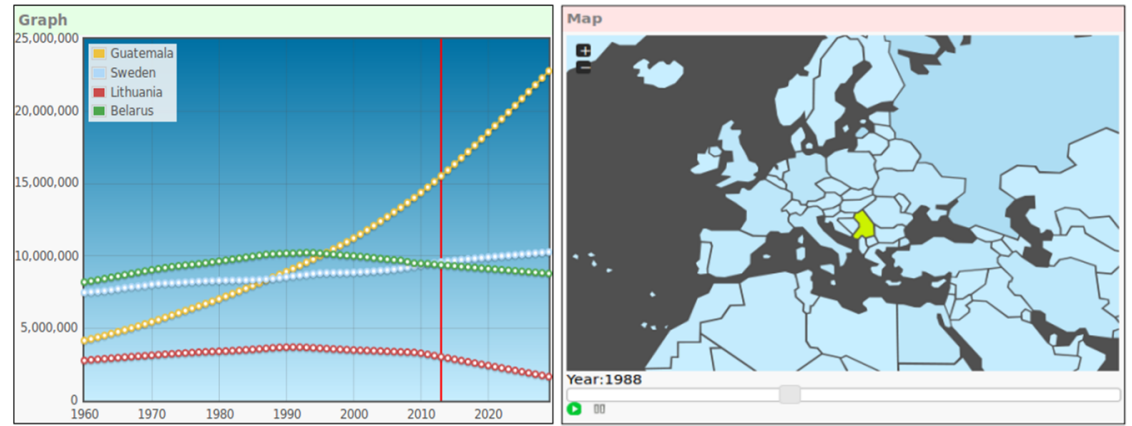

Figure 3: Output in early warning tool.

performed by commercial search engines. An example for national security purposes might be screening the web for pages and articles on "civil unrest", "civil war", "riots", "demonstrations", or others. The results are then summarized, analyzed, and visualized for the user. Since the interest does not only lie in the relative or absolute prevalence of a topic but additionally in the ongoing activity, the tool developed also takes this factor into account. The application also allows for analyzing the quantity of published articles on a topic as well as the country of origin of the articles (Stutzki [14]; Hauschild et al. [9, p. 15]) (as shown in Figure 4).

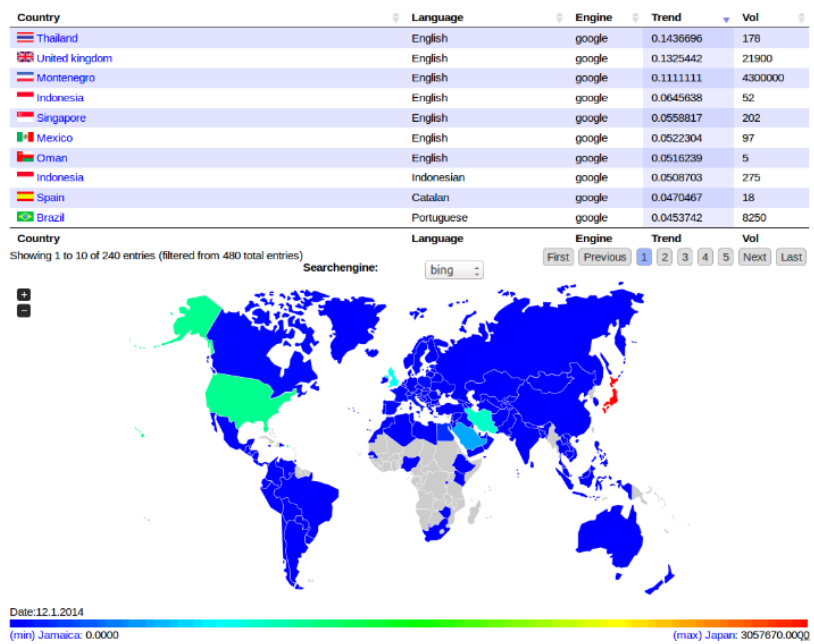

Figure 4: Output in topic monitoring tool.

This method might be very useful combined with Google Trends, a real-time index of the volume of queries that people enter into Google. Our tool does not provide this capability, since Google at the moment does not offer an API which would allow the integration. Especially with full access one can find querytrends in special geographical areas. Thus, the keywords from the trend detection 
will also be tested for being search engine queries at Google by a larger number of people. Using this tool is already successfully used for detecting economic trends (Choi and Varian [3]) and the outbreaks and spreads of diseases (Carneiro and Mylonakis [4]). Just like in the topic monitoring tool, detected keywords can be clustered based on their frequency on a geographical map.

Additionally, the use of further quantitative methods is recommended. The question of which methods may be applicable depends on the specific context of the risk management. The reason is, that a maximal possible number of data should be used in order to identify possible future threats. Weaknesses and limitations of single approaches should be compensated by using a selected set of different approaches.

The findings of these steps are finally analyzed using the qualitative method of expert interviews or focus groups, where a group of experts on the established content is confronted with the data gathered in the earlier steps as well as with the resulting geographical maps. On this basis the experts are discussing the results, summarize and evaluate the existing sample of threats and include them in the catalogue of hazards. The whole documentation and the catalogue of hazards are now passed into the next step of the process, the risk analysis.

\subsection{Risk analysis: define and analyze scenarios}

In the next step the decision maker creates possible future scenarios based on the existing data coming from the prior risk identification stage. Ideally, the expert from before support the scenario designing process. Thereby, the scenarios are firstly just collected on a broad range. Afterwards, the scenarios are ranked according to whatever criteria is needed. Furthermore, a ranking according to relevance and the uncertainty of occurrence is done, as the sum of all scenarios needs to be reduced. We developed the prototype of a software tool that detects the most relevant scenarios needing further analysis based on the experts' ratings. The advantage thereby is that the information is transferred without interruption to the next steps of analysis. Even if experts or decision makers change over steps, the information and data stays stored in the tool.

The scenarios being detected as relevant should be analyzed in more detail in a further step. Interconnections to several external influences as well as interconnections between scenarios might give additional insights into the scenario's network. Therefore, based on the approach of Vester [15] each scenario is illustrated in a network based on existing influences (positive $[+]$ and negative [-]). This also allows for getting additional insights into relevant reinforcing factors in the scenarios.

Hereby, the "Communication and Consultation" as well as the "Monitoring and Review" in the general risk management framework are of great importance, as newly identified impact factors should be further analyzed in following steps of analysis in the risk identification phase. Thus, in the next cycle of analysis e.g. additional keywords are to be analyzed in the tools used for trend detection. Furthermore, the network might be useful in the final step, the risk evaluation. Especially, if simulation is chosen to be the ideal tool for the risk evaluation. 


\subsection{Risk evaluation: a multi-model approach}

As the hazards were already detected and analyzed in detail, as well as relevant scenarios designed, the final step of the risk management tool deals with the evaluation of the scenarios. Therefore, the underlying findings and data from earlier steps need to be considered in order to decide, which approach fits best for an analysis. A unique approach for all scenarios is not estimated to deliver meaningful results, as the data basis for each scenario might differ significantly. Therefore, each scenario should be evaluated with a methodology that is able to handle the given case. For our research interest of national security and military threats we detected four different approaches that lead to meaningful results in the evaluation phase, statistical testing, system dynamics modeling, agent based modeling, and fuzzy-logic analysis.

If there is sufficient statistical data available, especially the statistical methods might be considered for evaluation and analysis. If the problem allows an analysis from the macro view and the prior conducted network analysis already shows meaningful insights, System Dynamics modeling might be chosen as shown. An application of a System Dynamics model can be found in (Hauschild et al. [9]) where it was used to analyze scenarios concerning the effects of the demographic change on the personnel structure and recruitment of the German Federal Armed Forces. If there is data on a micro level available and agents' actions can be estimated, Agent-Based modeling might be the most promising tool for evaluating the future scenarios and getting an idea of future likely changes. This model type is used to depict the behavior of autonomous agents, describing the potential actions and interactions between them and the environment. They are typically employed to analyze the collective, emergent behavior of the system under interest (Macal and North [12]). Agent-based models have been used in the social and political sciences for several years addressing topics as diverse as epidemics or ethnic conflicts (Epstein [5, p. xii]). (Hauschild et al. [9]) describes e.g. the development of an Agent-Based Model for analyzing social conflicts and unrest in Guinea. However, if the techniques presented so far cannot be used, a Fuzzy-Logic analysis might be conducted. Therefore, a list of several future scenario outcomes is developed and their relevance is weighted. In the field of application at hand, we might consider human, ecological, economic, supply security, and immaterial factors. Therefore, possible outcomes need to be defined, such as killed and injured population or the damage of living environment.

\section{Conclusion}

The use of OR-tools in (public) risk management and crisis management is widely accepted. However, especially the complexity of risk management for potential future threats demands a clearly structured process that guides the decision maker through various steps to a decision. Thus, risk management is a continuous process that does not deliver concrete values, but in general supports decision makers through showing possible scenarios and outcomes based on the 
underlying decisions made. Therefore we applied several tools and approaches to the ISO31000 normed risk management process and structured the decision making process based on three main steps, the identification of risks in a predefined context, the analysis of those risks based on possible scenarios, and finally the evaluation of the detected scenarios of interest and importance to the decision maker.

As the decision is - at best - just as good as the data available, especially the risk identifications stage plays a major role and combines several approaches. Therewith, we want to compensate limitations of single approaches and get a broad sphere of influencing factors. The qualitative approach allows for correcting implausible or unlikely trends in the data and adds another, very important view on the topic. However, the review and communication all through the risk management process is a key factor for success. Especially for combining the different methods and implementing a framework that considers learning effects from the different approaches used, further research is needed.

\section{Acknowledgement}

The support by the Planungsamt der Bundeswehr is gratefully acknowledged.

\section{References}

[1] Amanatidou E, Butter M, Carabias V, Könnölä T, Leis M, Saritas O, Schaper-Rinkel P, van Rij V (2012) On concepts and methods in horizon scanning: Lessons from initiating policy dialogues on emerging issues. Science and Public Policy 39(2): pp. 208-221.

[2] Buch, H. (2014) Interview with Heinrich Buch, Dipl. Pol., Oberst a.D., Senior Advisor at COMTESSA.

[3] Choi, H., Varian, H. (2011): Predicting the Present with Google Trends, Special Issue: Selected Papers from the 40th Australian Conference of Economists, Vol. 88, pp. 1-9.

[4] Carneiro, H.A., Mylonakis, E. (2009) Google Trends: A Web-Based Tool for Real-Time Surveillance of Disease Outbreaks, Surfing the Web, Vol. 49, pp. 1557-1564.

[5] Epstein, J. (2008): Generative Social Science. Studies in Agent-Based Computational Modelling. Princeton University Press.

[6] Federal Office for Civil Protection (2014) Integrated Risk Management, Bern, Switzerland.

[7] Goldstone, J.A. et al. (2005): A Global Forecasting Model of Political Instability, in Annual Meeting of the American Political Science.

[8] Habegger, B. (2010): Strategic foresight in public policy: Reviewing the experiences of the UK, Singapore, and the Netherlands, Futures 42, pp. 49-58.

[9] Hauschild, D., Leopold, A., Lohmann, S., Masala, C., Meyer-Nieberg, S., Pickl, S., Plenk, S., Tepel, T., Zsifkovits, M. (2014): Quantitative Methods of Future Studies, Final Report, Universität der Bundeswehr München. 
[10] ISO31000 (2009) Risk Management - Guidelines for principles and implementation of risk management.

[11] Masala, C., Pickl, S. (2013): Foresight Analysis: Quantitative Methoden der Zukunftsanalyse, Wehrwissenschaftliche Forschung - Jahresbericht 2013, pp. 58-59.

[12] Macal, C. M. and North, M. J. (2010). "Tutorial on agent-based modelling and simulation." Journal of Simulation 4(3): pp. 151-162.

[13] Palomino, M.A, T. Taylor, T. and Owen, R. (2012): Towards the Development of an Automated, Web-based, Horizon Scanning System, Proceedings of the Federated Conference on Computer Science and Information Systems, pp. 1009-1016.

[14] Stutzki, J. (2014): Multilingual Trend Detection in the Web, Proceedings of the 4th Student Conference on Operational Research SCOR 2014, OASICS, Vol. 37, pp. 16-24.

[15] Vester, F. (2000): Die Kunst vernetzt zu denken: Ideen und Werkzeuge für einen neuen Umgang mit Komplexität; DVA Stuttgart. 